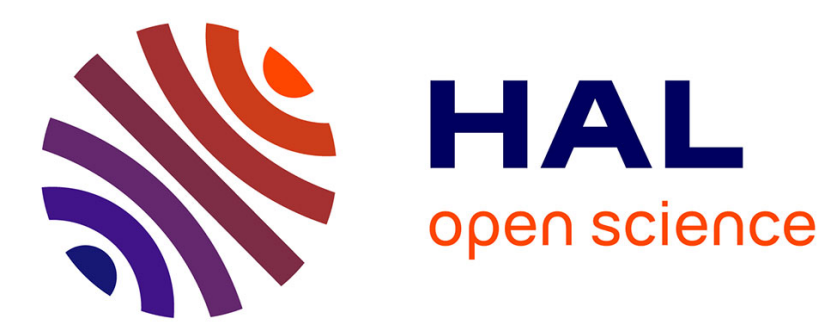

\title{
Electromagnetic interaction of vortices in layered superconducting structures
}

Alexandre I. Buzdin, Denis Feinberg

\section{To cite this version:}

Alexandre I. Buzdin, Denis Feinberg. Electromagnetic interaction of vortices in layered superconducting structures. Journal de Physique, 1990, 51 (17), pp.1971-1978. 10.1051/jphys:0199000510170197100 . jpa-00212502

\section{HAL Id: jpa-00212502 https://hal.science/jpa-00212502}

Submitted on 1 Jan 1990

HAL is a multi-disciplinary open access archive for the deposit and dissemination of scientific research documents, whether they are published or not. The documents may come from teaching and research institutions in France or abroad, or from public or private research centers.
L'archive ouverte pluridisciplinaire HAL, est destinée au dépôt et à la diffusion de documents scientifiques de niveau recherche, publiés ou non, émanant des établissements d'enseignement et de recherche français ou étrangers, des laboratoires publics ou privés. 
Classification

Physics Abstracts

$75.40-74.60-74.70 \mathrm{~J}$

\title{
Electromagnetic interaction of vortices in layered superconducting structures
}

\author{
A. Buzdin $\left({ }^{1}\right)$ and D. Feinberg $\left({ }^{2}\right)$ \\ (') Physics Department, Moscow State University, Moscow 117234, U.S.S.R. \\ ${ }^{2}$ ) Laboratoire d'Etudes des Propriétés Electroniques des Solides (*), Centre National de la \\ Recherche Scientifique, BP 166X, 38042 Grenoble, France
}

(Received on March 23, 1990, accepted in final form on May 7, 1990)

\begin{abstract}
Résumé. - On considère un système multicouches supraconducteur, avec un couplage entre couches d'origine strictement électromagnétique. On dérive une formule générale pour l'énergie d'une configuration arbitraire de vortex localisés dans les couches. On montre que l'énergie d'une paire vortex-antivortex sur la même couche possède une dépendance logarithmique avec la distance entre vortex pour une large séparation. La structure d'une ligne de vortex est d'autre part considérée pour un champ perpendiculaire. Deux régimes existent en fonction du rapport entre la distance intercouches $d$ et la longueur d'écran effective dans une seule couche $\lambda_{\text {eff }}$. Du fait de la dépendance en température de $\lambda_{\mathrm{L}}$, on s'attend à un crossover dans le comportement de $H_{\mathrm{c} 1}$.
\end{abstract}

Abstract. - A multilayered superconducting system with a purely electromagnetic coupling between layers is considered. A general formula is derived for the energy of an arbitrary configuration of point vortices localized in the layers. It is shown that the interaction energy of a vortex and an antivortex located on the same layer depends logarithmically on their distance for large distances. The structure of a vortex line in perpendicular fields is also considered. Two different regimes appear, depending on the ratio of the interlayer distance $d$ to the effective onelayer screening length $\lambda_{\text {eff }}=\lambda_{\mathrm{L}}^{2} / d_{0}$ where $\lambda_{\mathrm{L}}$ is the London screening length in the layers and $d_{0}$ the layer thickness. Due to the temperature dependence of $\lambda_{\mathrm{L}}$, the behaviour of the first critical field $H_{\mathrm{cl}}$ is expected to show a crossover below $T_{\mathrm{c}}$.

\section{Introduction.}

The peculiarity of vortices in superconducting films is connected with the fact that the superconducting current is flowing in the film whereas the magnetic field generated by the vortices exists in the whole space. As has been demonstrated by Pearl [1], the poor screening in the film leads to a specific dependence of the vortex energy with the film thickness $d_{0}$ and to a logarithmic interaction between vortices at distances $R<\lambda_{\text {eff }}=\lambda_{\mathrm{L}}^{2} / d_{0}$, where $\lambda_{\mathrm{L}}$ is the London penetration depth in the film. Only at distances larger than $\lambda_{\text {eff }}$ the

$\left(^{*}\right)$ Laboratoire associé à l'Université Joseph Fourier, France. 
interaction behaves like $1 / R$. As a result, for sufficiently thin superconducting films the Kosterlitz-Thouless phase may exist in the region near $T_{\mathrm{c}}$ [2]. There is also some evidence of a Kosterlitz-Thouless phase transition in layered high-temperature superconductors (see for example Refs. [3-5]).

In the case of layered superconductors there always exists an interaction between layers, through the magnetic field associated with the vortices. Thus the energy of vortices located in some given layer, as well as the interaction of vortices, will be modified as compared to the single layer case. The aim of this article is to calculate the energy of various vortex configurations in a layered superconducting system, the only coupling between layers being of electromagnetic origin. Notice that this model is quite suitable for describing the electrodynamics of artificially prepared superconducting superlattices [5] where the interlayer distance can be varied in a wide range. The electrodynamic coupling can indeed dominate the electronic coupling provided by Josephson tunneling when the layer separation is much larger than the transverse coherence length.

In section 2 we develop the general formalism within the frame work of the London approach. In section 3 we discuss the vortex-antivortex interaction, emphasizing the differences with the single layer case. In section 4 we consider the first critical field for multilayer superconductors and point out the possibility of a peculiar temperature dependence. In section 5 we discuss some aspects of thermal fluctuations of vortex lines.

\section{General formalism.}

We study here the case of type-II superconductors with a London penetration depth $\lambda_{\mathrm{L}}$ much larger than the coherence length $\xi$. We thus treat this situation within the framework of the London equations. In the case of non-interacting superconducting layers separated by $d$, one can generalize the London equation valid for a single layer $[1,6]$ (case of a thin film of thickness $d_{0}$ smaller than $\xi$ ) and write for any configuration of vortices

$$
\nabla^{2} \mathbf{A}=\frac{1}{\lambda_{\text {eff }}} \sum_{n}\left[\mathbf{A}-\Phi_{n}\right] \delta(z-n d)
$$

where $\mathbf{A}(\mathbf{r}, z)$ is the vector potential, $n$ is the layer index, $\Phi_{n}(\mathbf{r})=\sum_{\alpha} \varepsilon_{\alpha} \Phi\left(\mathbf{r}-\mathbf{R}_{n \alpha}\right)$ is the total London vector in layer $n$ and $\lambda_{\text {eff }}=\lambda_{\mathrm{L}}^{2} / d_{0}$ is the effective penetration depth in a single layer. The vector $\mathbf{R}_{n \alpha}$ denotes the position of the vortex $\alpha$ in layer $n$ and $\varepsilon_{\alpha}= \pm 1$ depending on its direction. The London vector $\Phi(\mathbf{r})$ for a single vortex is given in cylindrical coordinates $(r, \theta, z)$ by

$$
\Phi(\mathbf{r})=\left(0, \frac{\phi_{0}}{2 \pi r}, 0\right)
$$

and its Fourier transform in the $x y$ plane by

$$
\Phi(\mathbf{k})=i \phi_{0} \frac{[\mathbf{k}, \mathbf{z}]}{k^{2}}
$$

where $\phi_{0}=h c / 2 e, z$ is the unit vector along the direction perpendicular to the layers and $[a, b]$ stands from the vector product. Equation (1) corresponds to the situation where the density of current $\mathbf{j}$ is localized in the layers and homogeneous in each of them, defining the bidimensional current density $\mathbf{J}_{n}$ by

$$
\mathbf{j}(\mathbf{r}, z)=\sum_{n} \mathbf{J}_{n}(\mathbf{r}) \delta(z-n d) .
$$


It is important to underline that equation (1) gives the field distribution in full space. This distribution is itself determined by the current density $\mathbf{J}_{n}$, expressed by the London equation [7]

$$
\mathbf{J}_{n}(\mathbf{r})=\frac{c}{4 \pi \lambda_{\text {eff }}}\left[\Phi_{n}(\mathbf{r})-\mathbf{A}(\mathbf{r}, n d)\right]
$$

Due to the linearity of equation (1), one only needs to solve it for a single vortex, setting for instance $n=0, \mathbf{R}_{n \alpha}=0$. Performing the three-dimensional Fourier transform we have

where

$$
\tilde{\mathbf{A}}(\mathbf{k}, q)+\lambda_{\text {eff }}\left(k^{2}+q^{2}\right) \mathbf{A}(\mathbf{k}, q)=\sum_{n} \Phi_{n}(\mathbf{k}) \mathrm{e}^{-i q n d}
$$

$$
\begin{aligned}
\Phi_{n}(\mathbf{k}) & =\int \mathrm{d} \mathbf{r} \Phi_{n}(\mathbf{r}) \mathrm{e}^{-i \mathbf{k r}} \\
\mathbf{A}(\mathbf{k}, q) & =\int \mathrm{d} \mathbf{r} \mathrm{d} z \mathbf{A}(\mathbf{r}, z) \mathrm{e}^{-i(\mathbf{k r}+q z)} \\
\tilde{\mathbf{A}}(\mathbf{k}, q) & =\sum_{n} \int \mathrm{d} \mathbf{r} \mathrm{d} z \mathbf{A}(\mathbf{r}, z) \delta(z-n d) \mathrm{e}^{-i(\mathbf{k r}+q z)} .
\end{aligned}
$$

Expression (9) is easily transformed into

$$
\tilde{\mathbf{A}}(\mathbf{k}, q)=\frac{1}{\mathbf{d}} \sum_{n} \mathbf{A}(\mathbf{k}, q-n 2 \pi / d)
$$

which obviously verifies $\tilde{\mathbf{A}}(\mathbf{k}, q)=\tilde{\mathbf{A}}(\mathbf{k}, q-n 2 \pi / d)$ for any integer $n$. Using this property and the identity

$$
\sum_{n} \frac{1}{k^{2}+(q-n 2 \pi / d)^{2}}=\frac{d}{2 k} \frac{\sinh k d}{\cosh k d-\cos q d} .
$$

Equation (6) is easily solved, leading to the self-consistent equation

$$
\tilde{\mathbf{A}}(\mathbf{k}, q)=\frac{\sum_{n} \Phi_{n}(\mathbf{k}) \mathrm{e}^{-i q n d}-\tilde{\mathbf{A}}(\mathbf{k}, q)}{2 \lambda_{\text {eff }} k} \frac{\sinh k d}{\cosh k d-\cos q d}
$$

thus

$$
\tilde{\mathbf{A}}(\mathbf{k}, q)=\frac{\sum_{n} \Phi_{n}(\mathbf{k}) \mathrm{e}^{-i q n d}}{1+2 \lambda_{\text {eff }} k \frac{\cosh k d-\cos q d}{\sinh k d}} .
$$

The $2 \mathrm{~d}$ Fourier component of the vector potential in the $n$-th layer is

$$
\mathbf{A}_{n}(\mathbf{k})=d \int_{0}^{2 \pi / d} \frac{\mathrm{d} q}{2 \pi} \tilde{\mathbf{A}}(\mathbf{k}, q) \mathrm{e}^{i q n d}
$$

We finally obtain

$$
\mathbf{A}_{n}(\mathbf{k})=\sum_{m} \Phi_{m}(\mathbf{k}) \frac{\sinh k d}{2 \lambda_{\mathrm{eff}} k} \frac{\left(G_{k}-\sqrt{G_{k}^{2}-1}\right)^{|n-m|}}{\sqrt{G_{k}^{2}-1}}
$$


where the function $G_{k}$ is defined by

$$
G_{k}=\cosh k d+\frac{\sinh k d}{2 \lambda_{\text {eff }} k} .
$$

The total free energy in the London approximation is given by

$$
F=\frac{1}{2 c} \int \mathrm{d} \mathbf{r} \mathrm{d} z\left[\mathbf{A} \cdot \mathbf{j}+\frac{4 \pi \lambda_{\mathbf{L}}^{2}}{c} \mathbf{j}^{2}\right]
$$

The current being localized in the layers, a Fourier transform leads to

$$
F=\frac{1}{2 c} \sum_{n} \int \frac{\mathrm{d} \mathbf{k}}{(2 \pi)^{2}}\left[\frac{4 \pi \lambda_{\text {eff }}}{c}\left|\mathbf{J}_{n}(\mathbf{k})\right|^{2}+\mathbf{J}_{n}(-\mathbf{k}) \mathbf{A}_{n}(\mathbf{k})\right]
$$

where $\mathbf{J}_{n}(\mathbf{k})$ is the $2 d$ Fourier transform of $\mathbf{J}_{n}(\mathbf{r})$. Using (5) in Fourier transform, one finally obtains

$$
\begin{aligned}
F & =\frac{1}{2 c} \sum_{n} \int \frac{\mathrm{d} \mathbf{k}}{(2 \pi)^{2}} \mathbf{J}_{n}(-\mathbf{k}) \Phi_{n}(\mathbf{k}) \\
& =\frac{1}{8 \pi \lambda_{\text {eff }}} \sum_{n} \int \frac{\mathrm{d} \mathbf{k}}{(2 \pi)^{2}}\left[\left|\Phi_{n}(\mathbf{k})^{2}\right|-\Phi_{n}(-\mathbf{k}) \mathbf{A}_{n}(\mathbf{k})\right]
\end{aligned}
$$

Equation (19) combined with equation (15) provides the basis for the calculation of the free energy for various vortex configurations. In particular, the electromagnetic interaction energy between layers $n$ and $m$, due to the presence of vortices, is

$$
F_{\text {int }}(n-m)=-\frac{1}{4 \pi \lambda_{\text {eff }}} \int \frac{\mathrm{d} \mathbf{k}}{(2 \pi)^{2}} \Phi_{n}(\mathbf{k}) \Phi_{m}(-\mathbf{k}) \frac{\sinh k d}{2 \lambda_{\text {eff }} k \sqrt{G_{k}^{2}-1}}\left(G_{k}-\sqrt{G_{k}^{2}-1}\right)^{|n-m|}
$$

\section{Vortex-antivortex interaction.}

In contrast to the case of one single layer (thin film configuration), the energy of a single vortex in the presence of other layers is infinite. Expression (19) actually gives in this case

$$
F_{\mathrm{v}}=\frac{\phi_{0}^{2}}{16 \pi^{2} \lambda_{\mathrm{eff}}} \int \frac{\mathrm{d} k}{k}\left[1-\frac{\sinh k d}{2 \lambda_{\mathrm{eff}} k \sqrt{G_{k}^{2}-1}}\right]
$$

The upper cutoff is given by $k_{\mathrm{M}} \approx \xi^{-1}$. For finite $d$, this integral diverges logarithmically for small $K$, while for $d$ infinite (one layer case) one recovers the result of Pearl [1]

$$
F_{\mathrm{v}}^{0}=\frac{\phi_{0}^{2}}{16 \pi^{2} \lambda_{\mathrm{eff}}} \operatorname{Ln} \frac{\lambda_{\mathrm{eff}}}{\xi} .
$$

On the other hand, the energy $F_{\mathrm{v}-\overline{\mathrm{v}}}$ of a vortex-antivortex pair on the same layer is given by (19) with $\Phi_{n}(\mathbf{k})=\Phi_{\mathbf{k}}\left(1-\mathrm{e}^{i \mathbf{k} \mathbf{R}}\right)$, $\mathbf{R}$ denoting the vector linking the two vortices

$$
F_{\mathrm{v}-\overline{\mathrm{v}}}=\frac{\phi_{0}^{2}}{16 \pi^{2} \lambda_{\text {eff }}} \int \frac{\mathrm{d} k}{k} 2\left(1-J_{0}(k R)\right)\left[1-\frac{\sinh k d}{2 \lambda_{\mathrm{eff}} k \sqrt{G_{k}^{2}-1}}\right]
$$


where $J_{0}(z)$ is the first kind Bessel function. When $d \ll \lambda_{\text {eff }}$ we obtain from this expression

$$
F_{\mathrm{v}-\overline{\mathrm{v}}}=\frac{\phi_{0}^{2}}{16 \pi^{2} \lambda_{\text {eff }}} \int \frac{\mathrm{d} k}{k} 2\left(1-J_{0}(k R)\right)\left[1-\frac{1}{2} \sqrt{\frac{d}{\lambda_{\text {eff }}}}\right] \approx \frac{\phi_{0}^{2}}{8 \pi^{2} \lambda_{\text {eff }}} \operatorname{Ln} \frac{R}{\xi} .
$$

On the other hand, in the case $d \gg \lambda_{\text {eff }}$ we have practically the situation of independent layers. We obtain the result of Pearl, that is to say, for $R<\lambda_{\text {eff }}$ the interaction is logarithmic (see Eq. (24)) and for $R \gg \lambda_{\text {eff }}$, the interaction is of the order $\phi_{0}^{2} / 4 \pi^{2} R$ [1]. But within the limit of very large distance $R$ between vortices $(R \gg d)$ the free energy depends again logarithmically on the distance, although with a different prelogarithmic factor

$$
F_{\mathrm{v}-\bar{v}}=\frac{\phi_{0}^{2}}{4 \pi^{2} d} \operatorname{Ln} \frac{R}{d} \quad(R \rightarrow \infty) .
$$

In the case of layered high- $T_{\mathrm{c}}$ superconductors one has $d \ll \lambda_{\mathrm{eff}}$ and our calculation shows that the purely electromagnetic $v-\bar{v}$ interaction is always logarithmic at large distances. The situation is quite identical to that addressed by Kosterlitz and Thouless in their original work [8], contrarily to the thin film situation where for large distances it varies like $1 / R$. This conclusion is nevertheless correct only in the case of negligible electronic (Josephson) interaction between layers. For a full theory one needs to take this essential aspect into account. The previous discussion rather applies to the case of artificially synthetized layered superconducting structures where the Josephson coupling can be vanishingly small with respect to electromagnetic coupling. Let us make one more comment about $\mathrm{v}-\overline{\mathrm{v}}$ pairs where the two vortices do not lie in the same layer : in this case the integral giving the total energy diverges logarithmically as $k \rightarrow 0$, just as for a single vortex. This comes from the contribution of the two fields generated between the two layers containing the vortices, which do not cancel each other at large distances. One should not be worried with this result. Actually one verifies from (19) that a sufficient condition for the total energy of a system of vortices to remain finite is that the topological charge $\sum_{\alpha} \varepsilon_{n \alpha}$ is zero in each layer. This is consistent since, in the absence of any electronic coupling between layers, these configurations are the only ones which can be reached from a vortex-free state, by thermal fluctuations.

\section{First critical field for multilayered superconducting structures.}

Let us calculate the energy of a line of vortices perpendicular to the layers. This corresponds to the London vector $\Phi_{n}(k)=\Phi(k)$, i.e. the same in all the layers (Fig. 1). Equations (14) and (18) give after some transformations the energy per unit length

$$
F=\frac{\phi_{0}^{2}}{16 \pi^{2} \lambda_{\text {eff }} d} \int_{0}^{1 / \xi} \frac{\mathrm{d} k}{k+\frac{1}{2 \lambda_{\text {eff }}} \operatorname{coth} \frac{k d}{2}} .
$$

This expression can be written more conveniently in the following form, subtracting and adding the asymptotic $(k d \gg 1)$ form of the integrand

$$
F=\frac{\phi_{0}^{2}}{16 \pi^{2} \lambda_{\text {eff }} d}\left[\int_{0}^{\infty} \mathrm{d} x \frac{p(1-\operatorname{coth} x)}{(p x+\operatorname{coth} x)(p x+1)}+\int_{0}^{d / 2 \xi} \frac{p \mathrm{~d} x}{p x+1}\right]
$$

where $p=4 \lambda_{\text {eff }} / d$ and we hereafter assume $d \gg \xi$. 
a)

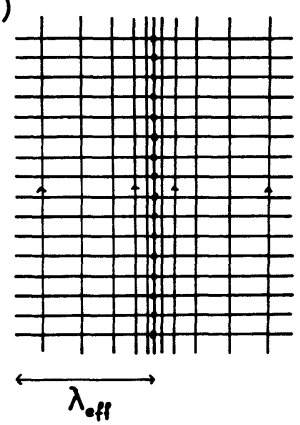

b)

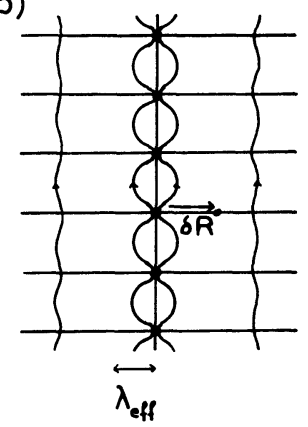

Fig. 1. - Line of vortices in a perpendicular field : a) strong coupling case $\left(d \ll \lambda_{\text {eff }}\right)$; b) weak coupling case $(d \gg \lambda$ eff $)$. The effect of a displacement $\delta R$ is calculated in section 5 .

In the limit $p \gg 1\left(d \ll \lambda_{\text {eff }}\right)$ expression (27) reduces to

$$
F=\frac{\phi_{0}^{2}}{16 \pi^{2} \lambda_{\text {eff }} d}\left[\operatorname{Ln} \frac{\lambda_{\text {eff }}}{\xi}+\frac{1}{2} \operatorname{Ln} \frac{1}{p}\right]=\frac{\phi_{0}^{2}}{16 \pi^{2} \bar{\lambda}^{2}} \operatorname{Ln} \frac{\bar{\lambda}}{\xi} \quad H_{\mathrm{cl}}=\frac{\phi_{0}}{4 \pi \bar{\lambda}^{2}} \operatorname{Ln} \frac{\bar{\lambda}}{\xi}
$$

where we define $\bar{\lambda}=\lambda_{\mathrm{L}} \sqrt{d / d_{0}}$ as an average London penetration depth. This result is quite natural since in this limit the magnetic field is practically uniform along the vortex axis (Fig. 1a).

In the other limit $p \ll 1\left(d \gg \lambda_{\text {eff }}\right)$ a similar analysis shows that one can neglect the first integral in equation (27), which yields

$$
F=\frac{\phi_{0}^{2}}{16 \pi^{2} \lambda_{\mathrm{eff}} d} \operatorname{Ln} \frac{\lambda_{\mathrm{eff}}}{\xi} \quad H_{\mathrm{cl}}=\frac{\phi_{0}}{4 \pi \bar{\lambda}^{2}} \operatorname{Ln} \frac{\lambda_{\mathrm{eff}}}{\xi}
$$

which corresponds to the energy of a vortex in a single layer. One verifies that the situation $p \ll 1$ corresponds to $\lambda_{\text {eff }} \ll \bar{\lambda}$. This means physically that screening occurs in each layer independently of the others (Fig. 1b).

Comparing expressions (28) and (29) one notices the different forms of the logarithmic factors. Whereas $\bar{\lambda} / \xi$ is temperature independent near $T_{\mathrm{c}}$, the ratio $\lambda_{\mathrm{eff}} / \xi=\lambda_{\mathrm{L}}^{2} / d_{0} \xi$ diverges at $T \approx T_{\mathrm{c}}$. This implies the existence of two different regimes for the temperature dependence of $H_{\mathrm{cl}}(T)$ for superlattices such as $d>\lambda_{\mathrm{eff}}(T=0)$. Above a characteristic temperature $T^{*}$ such as $d \approx \lambda_{\text {eff }}\left(T^{*}\right)$, expression (28) holds and leads to the usual dependence of $H_{\mathrm{cl}}(T)$, but a lower temperatures the slope of $H_{\mathrm{cl}}(T)$ must be decreased. This crossover occurs in the region where one would expect the usual linear temperature dependence for $H_{\mathrm{cl}}$. More precisely if $\lambda_{\mathrm{L}}(T)$ is given by the $\mathrm{G}$. L. expression close to $T_{\mathrm{c}}$ one obtains $T^{*} / T_{\mathrm{c}} \approx 1-\left(\lambda_{\mathrm{c}}^{2}(0) / 2 d d_{0}\right)$ (large $d$ case).

\section{Influence of thermal fluctuations on a vortex line.}

In order to check to what extent straight vortex lines may really exist, especially in the presence of thermal fluctuations, it is interesting to evaluate the rigidity of such a line. In the weak coupling case $\left(d \gg \lambda_{\text {eff }}\right)$, one can in a first step consider a very short scale deformation where one of the vortices forming the line is displaced in its layer from its equilibrium position by a distance $\delta R$ (Fig. 1b). 
Using the general formula (14) and (18), we obtain the energy of such a deformation

$$
\delta F=\frac{\phi_{0}^{2}}{16 \pi^{2} \lambda_{\mathrm{eff}}} \int \frac{\mathrm{d} k}{k} \frac{1-J_{0}(k \delta R)}{\sqrt{G_{k}^{2}-1}} \frac{\sinh k d}{2 \lambda_{\mathrm{eff}} k}\left[\sqrt{\frac{G_{k}+1}{G_{k}-1}}-1\right] .
$$

For $\delta R$ small compared to $d$, this leads to the following estimate

$$
\delta F \approx \phi_{0}^{2} \frac{\delta R^{2}}{d^{3}} \quad(\delta R \ll d) .
$$

For thermal fluctuations $\left\langle\delta R^{2}\right\rangle \approx T d^{3} / \phi_{0}^{2}$. Thus, for temperatures $T>T^{\prime} \approx \phi_{0}^{2} / d$, the fluctuation $\delta R$ is larger than $d$ and thermal fluctuations destroy short range ordering in the vortex lines.

Nevertheless, close enough to $T_{\mathrm{c}}$ (for $T>T^{*}$ ) one has necessarily $d<\lambda_{\text {eff }}$ and $\delta F$ goes to zero. A more careful analysis considering long range deformations is needed in this case, but one also expects a melting of flux lines. This question deserves further developments, in particular as regarding long range deformations.

\section{Conclusion.}

We have shown that even in the case where no electronic coupling exists between the superconducting layers, vortices which can be present due to external field or thermal fluctuations induce an electromagnetic coupling between layers. It results that the interaction between vortices in the same layer is influenced by the other layers, and the results notably differ from the single layer case (thin film). It is also different from the two-layer case treated in references [11] and [12]. A logarithmic dependence at large distances persists in this case. Nevertheless, for layered high- $T_{\mathrm{c}}$ superconductors the main task for an adequate description of the Kosterlitz-Thouless transition remains to take into account the Josephson coupling between layers. Reference [9] gives a negative answer to the question of the possibility of K. T. transition. As far as purely electromagnetically coupled layers are concerned, the situation is still not clear. One has like in quasi two-dimensional systems the possibility of coupling vortex-antivortex pairs in adjacent layers, forming small vortex loops [10]. These loops unfavor standard K. T. scaling. Here the electromagnetic interaction extends beyond adjacent layers and can have long range, rendering even more questionable the twodimensional behaviour. A careful discussion of screening in the multilayer situation is thus required. To this respect more information can be extracted from equations (19) and (20). Work is in progress in this direction.

We have calculated the first critical field in a superconducting superlattice. As far as its temperature dependence is concerned, two different regimes exist, depending on whether the layer spacing is small or large compared to the effective "single layer " London depth $\lambda_{\text {eff }}$.

The long period superconducting superlattices can be considered as a good model system for testing our analysis. They are also very suitable for investigating the vortex melting phenomena in perpendicular fields.

\section{Acknowledgments.}

One of us (A. B.) is grateful to Centre de Recherches sur les Très Basses Températures (CNRS) for warm hospitality during his stay in Grenoble. 


\section{References}

[1] Pearl J., Appl. Phys. Lett. 5 (1964) 65.

[2] Beasley M. R., Mooij J. E. and Orlando T. P., Phys. Rev. Lett. 42 (1979) 1169.

[3] Stamp P. C. E., Forrò L. and Ayache C., Phys. Rev. B 38 (1988) 2847 ;

See also Stamp P. C. E., Phys. Rev. Lett. 63 (1989) 582 : this comment refers to unpublished results on the same topic.

[4] Martin S., Fiory A. T., Fleming R. M., Espinosa G. P. and Cooper A. S., Phys. Rev. Lett. 62 (1989) 677.

[5] Artemenko S. N., Gorlova I. G. and Latishev Yu. I., Pis'ma Zh. Eksp. Teor. Fiz. 49 (1989) 566 ; Phys. Lett. A 138 (1989) 428.

[6] Jin B. Y. and Ketterson J. B., Adv. Phys. 38 (1989) 189.

[7] De Gennes P. G., Superconductivity of Metals and Alloys (Benjamin, New York) 1966, p. 60.

[8] Kosterlitz J. M. and Thouless D. J., J. Phys. C 6 (1973) 1181.

[9] Friedel J., J. Phys. France 49 (1988) 1561.

[10] Hixami S. and Tsuneto T., Prog. Theor. Phys. 63 (1980) 387.

[11] Sherill M. D., Phys. Rev. B 7 (1973) 1908.

[12] Clem J. R., Phys. Rev. B 12 (1975) 1742. 